\title{
Future Applications of ISFETs*
}

\author{
P. BERGVELD
}

University of Twente, Box 217, 7500AE Enschede (The Netherlands)

\begin{abstract}
The application of ISFETs has been focused up to now mainly on static measurements, comparable with the conventional way of applying ion-selective electrodes. The specific and unique properties of ISFETs, their small dimensions and very fast response, are not exploited explicitly in the case of static measurements. These features will, however, be explored more and more in the future, for example in flow-through and FIA systems as well as for ISFETs with an integrated coulometric actuator. In the latter case the ISFET is part of an active microchemical reactor. Similar systems may in the future contain bioactive materials, whose affinity to a biological analyte can be measured by means of stimulus-response experiments.
\end{abstract}

\section{Introduction}

Relevant future applications of ISFETs cannot be discussed without considering the original research, development and application aims and the corresponding results as achieved up to now.

The author's original intention in developing the ISFET was to measure the ionic sodium influx and potassium efflux at nerve and muscle membranes $[1,2]$. The silicon dioxide of a MOSFET, from which the gate metal was etched away, appeared to be sensitive for sodium and potassium ions, and thus well suited for the intended application. Sensitivity to $\mathrm{pH}$ was also observed, but was not

*Invited paper.

$0925-4005 / 91 / \$ 3.50$ seen as directly of interest for the electrophysiological experiments. The non-selectivity of silicon dioxide has recently been confirmed by independent measurements [3].

With respect to the measurement of ion fluxes, it should be noted that the related current densities are in the order of some $\mathrm{mA} / \mathrm{cm}^{2}$, resulting in ionic transports in the order of picomoles $/ \mathrm{cm}^{2}$. However, by catching the ionic flux in a very small cavity, which is achieved by pressing the ISFET with its surrounding ring of encapsulant firmly against the nerve membrane, the ionic changes may rise to $0.1 \mathrm{millimol} / \mathrm{litre}$, easily detectable by an ISFET. The recorded monophasic pulses [4], as shown in Fig. 1, are therefore most probably indeed the result of very local ionic concentration changes, although this has never been proved in any later paper.

In the context of the present paper, it is important to notice that in the electrophysiological ISFET application as mentioned above, the fast response of an ISFET is explicitly exploited, combined with its small dimensions.

In another, rather isolated paper by the present author, a chemically insensitive device has been described, related to the earlier ISFETs [5]. These devices were intended to be ISFETs, but, probably due to contamination of the gate oxide by a very thin epoxy layer, arising from the encapsulation of the devices, no sensitivity to ions was observed, at least not for an initial period of contact with the extracellular liquid. The device operated as a purely capacitive electrode, similar to a MOSFET. Because the only difference from the MOSFET was the omission of the gate metal, this device was called an OSFET.

(C) Elsevier Sequoia/Printed in The Netherlands 


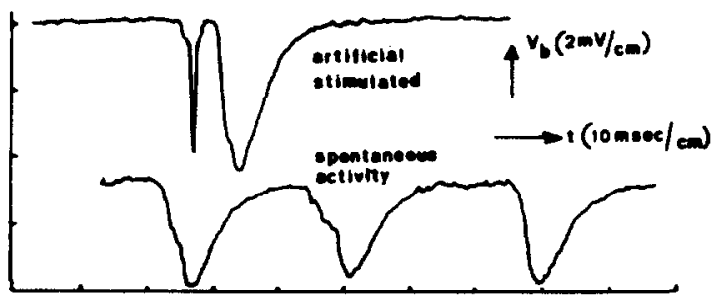

Fig. 1. Original registration of monophasic pulses with an ISFET pressed to the Locusta flexor tibialis.

Nowadays such a device would be called a REFET. However, the original OSFET differed from present REFETs in that the gate was surrounded by a relatively large parasitic gate. The result was that extracellular action potentials could be measured from a muscle fibre which contacted the actual gate as well as the parasitic gate. In fact the potentials as caused by extracellular ionic currents were measured by means of a capacitive division between the actual gate capacitance and the parasitic gate capacitance. The recordings excelled in a very good signal-to-noise ratio as compared to the usual recordings with metal microelectrodes and also the broad frequency range $(0<f<45 \mathrm{kHz})$ was remarkable. Unfortunately the excellent properties of recording features as mentioned above have never been exploited since its first publication, although recently the possibilities have been rediscovered [6].

In view of the present paper it should be noted that the parasitic gate in fact operated as a capacitive reference electrode. Due to its size, its capacitance was much larger than the gate capacitance which in fact acted as a micro-electrode. The capacitive voltage division therefore resulted in a nearly unattenuated conversion of the extracellular potentials. It should be kept in mind that a capacitive reference might in the future also be explored, especially with respect to dynamic ISFET applications such as those described below.

\section{From Static to Dynamic Measurements}

The follow-up of the initial work, as briefly summarized in the previous Section, was not directed towards the electrophysiological application of ISFETs and related devices, but to the measurement of ionic constituents of various body liquids, mostly blood. This was due to the lack of any small conventional type of ion-selective electrode suitable for in vivo application. This is still the reason that many ISFET projects at University as well as industrial laboratories are devoted to extra- or intracorporeal measurements of blood chemical constituents.

Application in analytical chemistry is less pursued, because of the apparent adequacy of conventional ion-selective electrodes and other analytical methods. This may, however, change in the future as soon as the specific properties of ISFETs are more clearly recognized, such as their extremely short response time, which is of great advantage in the case of flow injection analysis (FIA) and other microchemical investigations, for example.

Although the electrophysiological experiments, as mentioned in the Introduction, already showed from the beginning that ion fluxes occurring within milliseconds could easily be recorded, this fact was hardly recognized at that moment. The reason is most probably that directly after the first description of ISFETs, these devices were seen as a modern solid-state appearance of the more bulky conventional (glass) membrane electrodes and thus should replace this type of electrode. Much effort was and still is therefore spent on static measurements with the drawbacks of drift and lack of a stable solidstate reference electrode. The benefits of these investigations are the gain in knowledge of the actual ISFET operation and the application of better inorganic gate materials, such as $\mathrm{Al}_{2} \mathrm{O}_{3}, \mathrm{Si}_{3} \mathrm{~N}_{4}$ and $\mathrm{Ta}_{2} \mathrm{O}_{5}$. Nevertheless it is still doubtful, regardless of further improvements in ISFET technology, whether it will ever be possible to prepare drift-free ISFETs. The reason is that any device with an insulating layer exposed to an electric field will drift, as long as mobile charges in the insulator cannot be abandoned completely. In this respect ISFETs can hardly compete with their conventional counterparts, whose operation 
relies on ion exchange mechanisms at conducting membranes [7]. Furthermore a stable solid-state, liquid-free reference electrode is still not developed.

More successful applications of ISFETs can, in the opinion of the author, sooner be expected in the area of dynamic measurements than in the competitive field of static measurements. The first examples are already given in the literature and will be reviewed in the following Sections. Not only are the specific ISFET properties, namely small dimensions and fast response, more explicitly explored, but also the reference problem can be solved much more easily in the case of dynamic measurements.

\section{Application of ISFETs in Flow-through Cells}

The fast response of an ISFET has already been exploited in flow-through cells, where a sample is injected into a carrier stream, as schematically drawn in Fig. 2.

The first system based on this approach was published in 1980 [8], while Sibbald introduced it for on-line clinical analysis of blood ionic composition, using a flowthrough cell with four integrated membranecovered ISFETs [9]. Especially adapted to the flow-through cell configuration, backside contacted ISFETs can be used with great advantage for this type of measurement, as recently shown by Alegret [10].

In all the examples mentioned above, the reference electrode consisted of a conven-

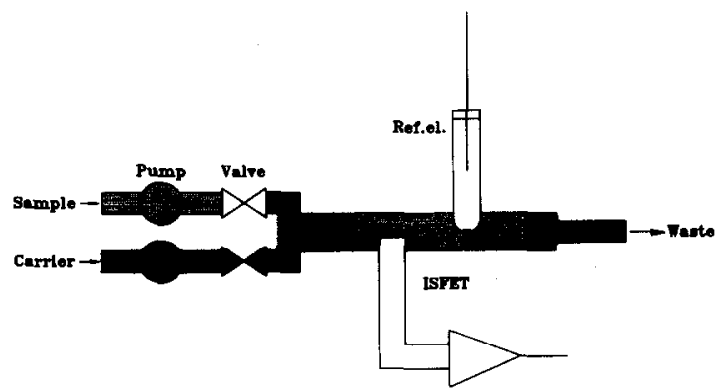

Fig. 2. Schematic representation of single ISFET flowthrough cell arrangement. tional liquid-filled reference electrode, with the well-known problems of the liquid junction potential which can also react very fast to a change in ionic composition, this potential change possibly coinciding with the ISFET response to the sample. Moreover, especially if the sample is whole blood, contamination of the reference electrode frit cannot be excluded. Only the use of heparinized calibration (and rinsing) liquids can prevent this contamination, but it makes the system more complicated [9]. A solution to the reference problem is the application of a differential measuring set-up, as described in the following Sections.

\section{Differential Measurements}

To solve the reference electrode problem, a differential measurement set-up has been proposed [11] in which case the ISFET response is measured with respect to a REFET [12], while the analyte potential is defined by a platinum wire, as shown schematically in Fig. 3.

The unstable electrolyte/platinum electrode potential is a common signal to the differential system and will thus not interfere with the final output signal. Therefore the platinum electrode is usually called a pseudoreference electrode.

However, for static measurements such systems require that the REFET is at least as stable as the ISFET, while furthermore the electrical characteristics of the ISFET and

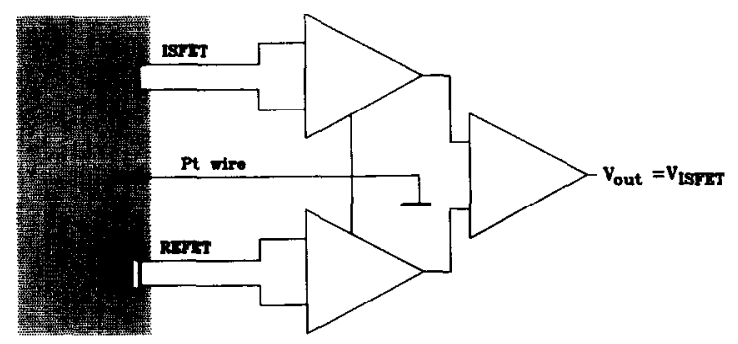

Fig. 3. ISFET/REFET differential measurement with platinum pseudo-reference electrode. 
REFET should be identical. The development of REFETs which can meet these requirements is still in its infancy, especially for cases where the analyte contains an unknown composition of many types of ions. For dynamic measurements the REFET can, however, be replaced by an ISFET which is temporarily in contact with a constant $\mathrm{pH}$; this condition can be fulfilled in a flow-through cell.

\section{Flow-through Cell with Two Identical ISFETs}

Differential measurements as proposed in the previous Section can be performed with a flow-through system as schematically shown in Fig. 4.

If only the carrier solution is pumped along the two ISFETs, the differential signal should be zero. If not, the difference can be set to zero. After injection of the sample solution, ISFET ${ }_{1}$ will first sense the sample, while ISFET $_{2}$ is still in contact with the carrier solution, thus acting at this moment as a pseudo-REFET. The differential signal thus reflects the sample concentration with respect to the carrier concentration. As soon as the sample contacts both ISFETs, the differential signal will again be zero. After the sample leaves ISFET $_{1}$, this ISFET will be the pseudo-REFET and ISFET ${ }_{2}$ will measure the sample with respect to the carrier solution. The signal will then be reversed in polarity, but will show the same amplitude as the first signal.

A positive point of this type of flowthrough cell is that two identical sensors can be used, but a negative point is that they cannot be integrated in one chip. The distance between the two ISFETs should be

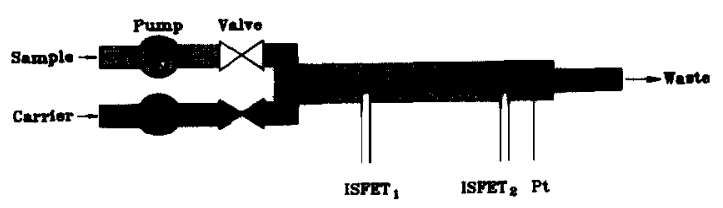

Fig. 4. Flow-through cell with two identical ISFETs and pseudo-reference electrode. such that one of the devices should become in equilibrium with the sample solution and that during this period the other should continuously be in contact with the carrier solution. This requirement will generally result in relatively long distances between the two separate chips.

This type of flow-through cell is explored by Gumbrecht $e t$ al. for the measurement of blood samples in a bedside unit, where blood samples and calibration solution are carried to the cell by means of a double lumen needle [13].

A similar system for the measurement of urea is applied by Chandler et al., where between the two identical ISFETs an enzyme column is mounted, in this particular case loaded with urease [14]. Urea injection results in measured peaks (differential), whose amplitude is proportional to the concentration of urea.

\section{ISFET with Retarded Response as Pseudo-REFET}

As already mentioned, a maximum signal in the flow-through cell, as described in the previous Section, can only be achieved if the distance between the two ISFETs is relatively large. This distance can be decreased in the case that the response time of the second ISFET has been artificially increased by adding a membrane which limits the diffusion of ions. This system is shown in Fig. 5.

In contrast to the system shown in Fig. 4, both ISFETs can now be in contact with the sample solution at the same time. The second ISFET should be slow enough to achieve a maximum signal with the first ISFET before

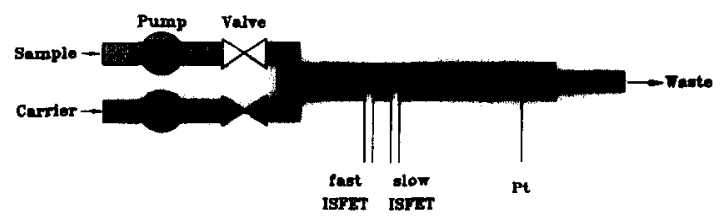

Fig. 5. Flow-through cell with one fast ISFET and one slow ISFET (retarded response) and pseudo-reference electrode. 
the second ISFET starts to respond. A drawback of this approach may be that the intersample time now also increases, because the membrane of the second ISFET should be washed after each sample, limiting the number of samples per unit of time.

The system is described in the literature by van den Vlekkert et al., using a polyHEMA layer of $200 \mu \mathrm{m}$ for the pseudo-REFET, resulting in a response delay of $200 \mathrm{~s}$ [15]. This is sufficiently low as compared to the time constant $(10 \mathrm{~s})$ of the membrane-covered ISFET ion sensors $\left(\mathrm{K}^{+}\right.$and $\left.\mathrm{Ca}^{++}\right)$in the flow-through cell.

Although van den Vlekkert et al. use separate chips for the ion sensors and the pseudoREFET, it is technologically not difficult to integrate the devices into one chip. Using micromachining techniques it might be possible that in the future a sample inlet valve will also be integrated.

\section{Flow Injection Titration Systems}

Volumetric acid/base titration is a wellknown technique to determine the acidity of a sample by means of adding a certain amount of base or acid to the sample and to detect the so-called equivalence point in the titration curve. This is the point where the added reagent exactly neutralizes the sample. For high-speed titrations the $\mathrm{pH}$ of the mixture will change very rapidly and therefore a fast $\mathrm{pH}$ sensor, such as the ISFET, is a very attractive detector. Wang et al. showed the usefulness of an ISFET by injecting a $40 \mu 1$ sample via a gradient chamber in a titrant solution, which flows continuously along an ISFET and a conventional reference electrode, placed directly beyond the ISFET [16]. The system is schematically shown in Fig. 6.

The concentration gradient of the sample passes the ISFET twice, so the equivalence point between sample solution and titrant is also encountered twice during one titration cycle. The time span between the two equivalence points is used to determine the sample concentration and this parameter appeared to

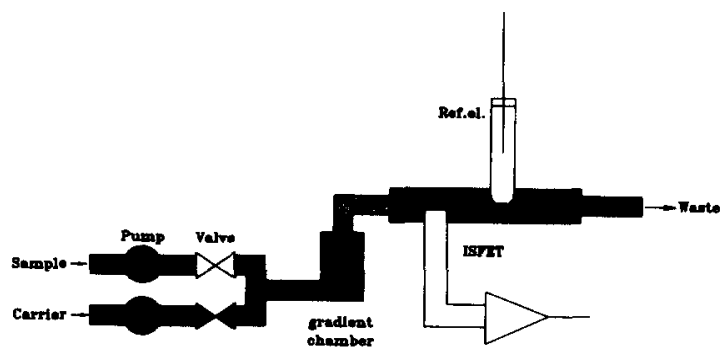

Fig. 6. Schematic representation of flow injection volumetric titration system.

be proportional to the concentration of the sample.

Comparing this FIA titration method with the different flow-through methods described in the previous Sections, it should be noted that now not the peak height but the peak width of the response curves is the actual signal which provides the analytical data. This reduces the requirements of stability and reproducibility of the applied ISFETs.

It will be clear that for the detection of the occurring $\mathrm{pH}$ changes, in the order of 10 to $30 \mathrm{~s}$, an ISFET is an attractive type of sensor. This is even more the case with a coulometric titration, when the titrant is generated at an adjacent electrode by means of coulometry. Especially with small samples, this technique results in very fast titrations, as will be shown in the next Section.

\section{Coulometric Titrations}

Van der Schoot et al. constructed for the first time a complete coulometric titrator in silicon [17].

The titration 'vessel' consisted of a cavity of $80 \mu \mathrm{m}$ depth, etched in a piece of silicon, containing also two etched holes, which is sealed on a planar array of ten ISFETs as schematically shown in Fig. 7.

Each of the ISFETs is surrounded by a thin-film gold electrode. The application of a current between two of these electrodes will result in the electrolysis of water after the cavity is filled via the holes with a sample solution from which the acid or base 


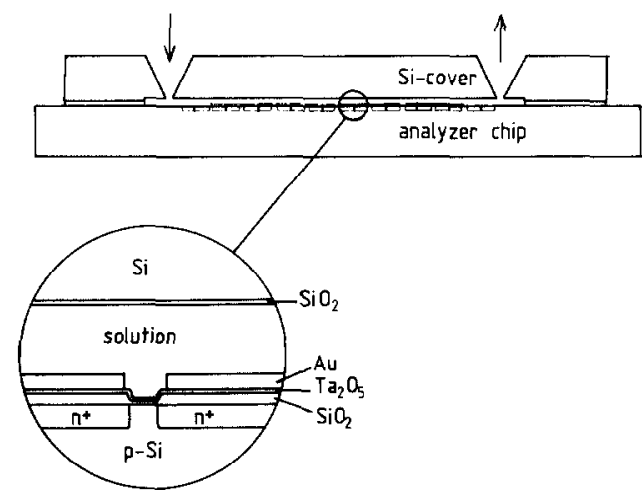

Fig. 7. Cross section of coulometric titration system.

concentration has to be determined. The electrolysis of water results in a local decrease of the $\mathrm{pH}$ at the anode and the reverse at the cathode. This local coulometric titration is measured by the ISFET inside the actuator electrode with respect to an ISFET which is not positioned in a titrated area. Note that in this case a normal ISFET acts as pseudoREFET, while one of the gold electrodes not applied for the $\mathrm{pH}$ generation is used as a pseudo-reference electrode. It appeared that the sample could be titrated in a few seconds, with the ISFET acting only as an indicator to determine the equivalence point of the coulometric titration. It will be obvious that the fast response, small dimensions, as well as the planar performance of the ISFET are in this example in fact the key features of the technique.

In the dynamic measurement mentioned above the ISFET is no longer used in a static way and the d.c. value of the ISFET amplifier output voltage is no longer of importance. Instead the elapsed time between the onset of the actuator current and the detection of the equivalence point is measured. This titration time is proportional to the acid or base concentration of the sample. Possible ISFET drift or sensitivity degradation does not influence the measurement results.

The same titration experiments are also possible in an open vessel with a dip-stick system, provided that the liquid is unstirred [18]. As long as the mass transfer is diffusion controlled, now the square root of the titra- tion time is proportional to the acid or base concentration of the sample.

By providing the system shown in Fig. 7 with an additional hole in the silicon cover, over which a Teflon membrane is deposited, a $\mathrm{CO}_{2}$ sensor has been created [19]. Because the acidity of an internal bicarbonate solution will be a function of the external $\mathrm{CO}_{2}$ pressure, this pressure could be determined by applying continuously forward and back titration cycles. The system could measure, after one initial calibration, for at least 50 days without recalibration, resulting in a drift of $10 \%$. This is in terms of $\mathrm{pH}$ only 0.001 units per day, which is a great improvement compared with a 'normal' ISFET drift of $0.02 \mathrm{pH} / \mathrm{h}$.

\section{Ion-step Experiments with ImmunoFETs}

It has long been debated whether it would be possible to measure the charge redistribution that is characteristic for an antibody/ antigen interaction with an ISFET, if first the antibodies are immobilized on the surface and then the created IMFET is incubated with the corresponding antigens. Nowadays it is recognized that this is not possible, because, although proteins are charged molecules, the counter charges are in the direct vicinity, preventing them from generating a static external electric field. Nevertheless, the existence of fixed charges of proteins, immobolized in a layer onto an ISFET surface, can be detected by using a dynamic method of measuring. For that, the proteincovered ISFET should be exposed to a sudden change in electrolyte concentration [20]. This process is schematically represented in Fig. 8.

After a stepwise increase of the electrolyte salt concentration, usually $\mathrm{KCl}$, the ions will diffuse into the protein membrane, but not with the same speed, due to a difference in mobility caused by the presence of the fixed charges. This means that the initially constant output voltage of the ISFET amplifier shows a temporary disturbance. The amplitude of 

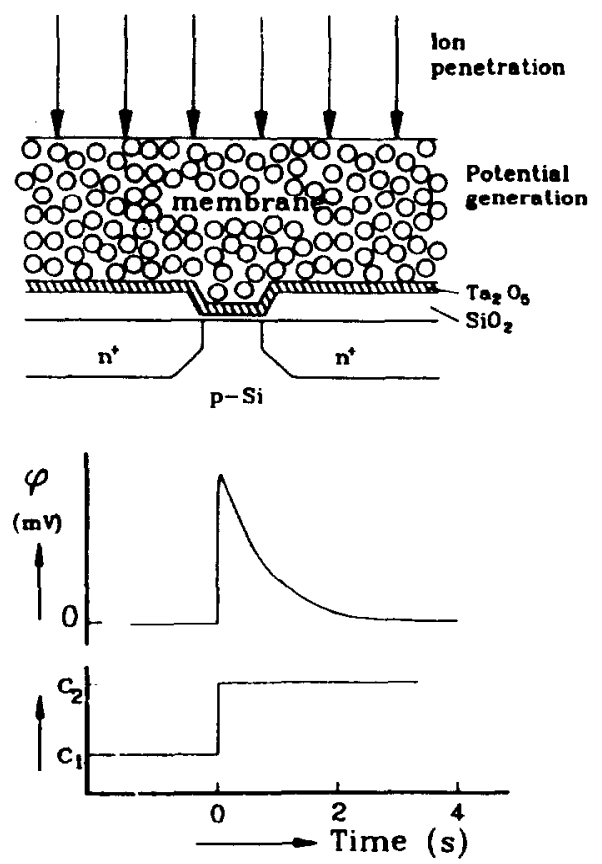

Fig. 8. Schematic representation of immunoFET to which the ion step procedure is applied.

the pulsatile membrane voltage appears to be a function of the total effective fixed membrane charge. This could be proved by gradually changing the $\mathrm{pH}$ of the solution and in the meantime carrying out the ion step procedure at regular intervals. Because proteins are amphoteric molecules, the protein charge will change as a function of the changing $\mathrm{pH}$. Consequently a point, the so-called isoelectric point, will be passed where the proteins are uncharged. At this point the amplitude of the transient membrane potential indeed appeared to be zero. Before or beyond this point the amplitudes were positive or negative, due to the corresponding negative or positive protein charge at that specific $\mathrm{pH}$. In this way the difference in isoelectric points of antibodies, adsorbed to a porous membrane which was deposited onto the ISFET surface, and the subsequently formed antibody/antigen complexes could be detected.

Although the method has still to be evaluated and further developed to achieve an IMFET which can be used practically for the direct monitoring of an immunological reaction, it will be clear that the method is based on the measurement of induced transient membrane potentials, occurring within seconds, requiring a sensor with a fast response. Again in this case the ISFET is an excellent candidate, where the sensitivity for electrical potentials, superimposed on a relatively stable baseline, is the only requirement. It is striking that in fact this is the same requirement as for the original electrophysiological experiments mentioned in the Introduction.

\section{Conclusions and Future Outlook}

\section{Flow-through Cells}

With respect to the different flow-through cells described in the first Sections, it should be mentioned that these type of measurements are often called flow-injection analysis (FIA) experiments, because a small sample is injected into a carrier stream. However, all systems described in the literature up to now make use of equilibrium measurements between the sample solution and the ISFET, while real FIA systems employ non-equilibrium data [21]. These measurements require however, that the calibration and sample solutions have the same invariant viscosity. Furthermore, the hydrodynamics of the injection valve and the analysis cell, including the applied tubing, as well as the response time of the sensor should remain constant during the experiment. Only when these requirements are fulfilled can non-equilibrium data also be used for the chemical analysis of the sample, like peak height, peak width at selected level and peak area. Maybe in the future these requirements can be met by constructing the complete FIA system in silicon as an integration of ISFET, valves and channels by means of micromachining.

\section{Microlitre Titrations}

It should be noted that the coulometric microlitre titration experiments are limited to the measurement of acid-base concentrations as long as no solid-state actuators exist which can generate or consume ions other than protons, to be measured by corresponding 
ion sensors. The exploitation of other electroactive materials, such as Prussian Blue [22], may change this in the future, although no experience exists at the present moment with respect to actuator properties of this type of materials.

In the microlitre titration vessel [17] and the dip-stick titrator [18], the actuator was shaped around the ISFET gate. Generated protons or hydroxyl ions have therefore to diffuse over a certain distance, typically $10 \mu \mathrm{m}$, before the ISFET will respond. This limits the speed of titration. As soon as the distance between the ISFET and the actuator is decreased, for example, by applying a porous electrode over the gate of an ISFET, it can be expected that the speed of titration will be enlarged, in which case the fast response time of an ISFET is again very favourable.

By ultimate integration of ISFET and actuator in the near future, a new area of microchemical analysis may come into existence.

\section{Ion Step Experiments}

The new approach of dynamic measurements with ImmunoFETs is at present a first example of a stimulus-response type of measurement. The ion step induces a membrane diffusion potential due to the difference of ion mobilities of cations and anions caused by the net charge density of the biomolecules inside the membrane. The thickness of the membrane has been chosen relatively arbitrarily around $5 \mu \mathrm{m}$, resulting in pulses with a duration of seconds. Optimization of the procedure may however result in much thinner membranes, which are necessary to decrease the incubation time, for example, leading to much faster responses. The application of an ISFET guarantees in this case that these pulses can also be measured.

\section{Reference Electrodes}

In the flow-through cell experiments it was shown that the conventional liquid-filled reference electrode can be replaced by solid-state electrodes like REFETs or pseudo-
REFETs. A REFET is in fact a capacitive type of electrode, applied to measure the electrical potential of the electrolyte. In the electrophysiological experiments mentioned in the Introduction [5], the capacitive reference electrode consisted of a parasitic gate surrounding the actual gate of the measuring FET. In this way voltage pulses in the millisecond range could easily be detected. Especially in the case of dynamic measurements, which result in ever shorter pulses, this approach may in the future again be worth considering.

\section{References}

1 P. Bergveld, Development of an ion-sensitive solid state device for neurophysiological measurements, IEEE Trans. Biomed. Eng., BME-17 (1970) 70-71.

2 P. Bergveld, Development, operation and application of the ion-sensitive field effect transistor as a tool for electrophysiology, IEEE Trans. Biomed. Eng., BME-19 (1972) 342-351.

3 S. C. Chen, Y-K. Su and J. S. Tzeng, The fabrication and characterisation of ISFETs with silicon dioxide gate, J. Phys. D: Appl. Phys., 19 (1986) 1951-1956.

$4 \mathrm{P}$. Bergveld, Ion sensitive field effect transistor, Proc. Conf. Biocapt '75, Vol. 1, Paris, 1975, pp. 299-304.

5 P. Bergveld, J. Wiersma and H. Meertens, Extracellular potential recordings by means of a field effect transistor without gate metal, called OSFET, IEEE Trans. Biomed. Eng., BME-23 (1976) 136-144.

6 A. Cambiaso, M. Grattarola, G. Arnaldi, S. Martinoia and $G$. Massobrio, Detection of cell activity via ISFET devices; modelling and computer simulations, Sensors and Actuators B, 1 (1990) 373-379.

7 P. Bergveld, Exploiting the dynamic properties of FET-based chemical sensors, J. Phys. E: Sci. Instrum., 22 (1989) 678-683.

8 A. U. Ramsing, J. Janata, J. Ruzicka and M. Levy, Miniaturization in analytical chemistry - a combination of flow injection analysis and ion-sensitive field effect transistors for determination of $\mathrm{pH}$, and potassium and calcium ions, Anal. Chim. Acta, 118 (1980) 45-52.

9 A. Sibbald, P. D. Whalley and A. K. Covington, A miniature flow-through cell with a four-function integrated circuit for simultaneous measurements of $\mathrm{K}^{+}, \mathrm{H}^{+}, \mathrm{Ca}^{++}$, and $\mathrm{Na}^{+}$ions, Anal. Chim. Acta, 159 (1984) 47-62.

10 S. Alegret, J. Alonso, J. Bartroli and J. Domenech, Flow-through pH-ISFET detector for flow injection analysis, Anal. Chim. Acta, 222 (1989) 373-377. 
11 P. Bergueld, A. van den Berg, P. D. van der Wal, M. Skowronska-Ptasinka, E. J. R. Sudhölter and D. N. Reinhoudt, How electrical and chemical requirements for REFETs may coincide, Sensors and Actuators, 18 (1989) 309-327.

12 M. Skowronska-Ptasinska, P. D. van der Wal, A. van den Berg, P. Bergveld, E. J. R. Sudhölter and D. N. Reinhoudt, Reference field effect transistors based on chemically modified ISFETs, Anal. Chim. Acta, 230 (1990) 67-73.

13 W. Gumbrecht, W. Schelter, B. Montag, M. Rasinski and U. Pfeiffer, On-line blood electrolyte monitoring with a CHEMFET microcell system, Sensors and Actuators B, 1 (1990) 477-480.

14 G. K. Chandler, J. R. Dodgson and M. J. Eddowes, An ISFET-based flow injection analysis system for determination of urea; experiment and theory, Sensors and Actuators B, $I$ (1990) 433-437.

15 H. H. van den Vlekkert, N. F. de Rooij, A. van den Berg and A. Grisel, Multi-ion sensing system based on glass-encapsulated pH-ISFETs and a pseudoREFET, Sensors and Actuators B, 1 (1990) 395-400.

16 G. H. Wang, D. Yu and Y. Sun, pH-ISEFT used as a detector in a flow injection titration system, Sensors and Actuators, 19 (1989) 41-52.
17 B. H. van der Schoot and P. Bergveld, An ISFETbased microlitre titrator: integration of a chemical sensor-actuator system, Sensors and Actuators, 8 (1985) 11-22.

18 W. Olthuis, B. H. van der Schoot, F. Chavez and P. Bergveld, A dip-stick sensor for coulometric acidbase titrations, Sensors and Actuators, 17 (1989) 279-284.

19 B. H. van der Schoot and P. Bergveld, Coulometric sensors, the application of a sensor-actuator system for long-term stability in chemical sensing, Sensors and Actuators, 13 (1988) 251-262.

20 R. B. M. Schasfoort, R. P. H. Kooyman, P. Bergveld and J. Greve, A new approach to ImmunoFET operation, Biosensors Bioelectron., 5 (1990) 103-124.

21 E. H. Hansen, Flow injection analysis-recent developments and future trends, in G. G. Guibault and M. Mascini (eds.), Analytical Uses of Immobilized Biological Compounds for Detection, Medical and Industrial Uses, Reidel, Dordrecht, 1988, pp. 291-308.

22 H. Hartman, E. W. Grabner and P. Bergveld, Prussian Blue-coated interdigitated array electrodes for possible analytical application, Anal. Chim. Acta., submitted for publication. 